Vol. 14, No. 2, June 2010, pp. 65-76

Invited paper

\title{
Development of Nanostructured Plasmonic Substrates for Enhanced Optical Biosensing
}

\author{
Kyung Min Byun* \\ Department of Biomedical Engineering, Kyung Hee University, Yongin 446-701, Korea
}

(Received May 25, 2010 : revised June 8, 2010 : accepted June 8, 2010)

\begin{abstract}
Plasmonic-based biosensing technologies have been successfully commercialized and applied for monitoring various biomolecular interactions occurring at a sensor surface. In particular, the recent advances in nanofabrication methods and nanoparticle syntheses provide a new route to overcome the limitations of a conventional surface plasmon resonance biosensor, such as detection limit, sensitivity, selectivity, and throughput. In this paper, optical and physical properties of plasmonic nanostructures and their contributions to a realization of enhanced optical detection platforms are reviewed. Following vast surveys of the exploitation of metallic nanostructures supporting localized field enhancement, we will propose an outlook for future directions associated with a development of new types of plasmonic sensing substrates.
\end{abstract}

Keywords: Surface plasmons, Localized surface plasmon, Biosensor, Metallic nanostructures, Nanofabrication

OCIS codes : (050.6624) Subwavelength structures; (220.4241) Nanostructure fabrication; (240.6680) Surface plasmons; (280.1415) Biological sensing and sensors; (310.0310) Thin films

\section{INTRODUCTION}

Surface plasmon resonance (SPR) technique has been successfully used in a variety of sensing applications, since it provides rapid, label-free, and quantitative sensing capability when detecting biomolecular reactions on a sensor surface $[1,2]$. SPR is attributed to the excitation of surface plasmon polaritons (SPPs) when a transverse magnetic (TM)polarized beam incident on a thin metal film between two dielectric media is coupled to surface plasmons formed in the film [3]. These plasmons resonantly couple with the incident light at a specific resonance angle when the momentum matching between an incident photon and a SPP is achieved. They propagate tens to hundreds of micrometers along the metal surface and also decay exponentially away from the surface. Across the interface between a metal film and a surrounding dielectric medium, the wave vector, $k_{\mathrm{SPP}}$, is continuous for momentum matching, so that the dispersion relation can be written as:

$$
k_{\mathrm{SPP}}=\frac{\omega}{c} \sqrt{\frac{\varepsilon_{\mathrm{M}} \varepsilon_{\mathrm{D}}}{\varepsilon_{\mathrm{M}}+\varepsilon_{\mathrm{D}}}}
$$

where $\omega$ and $c$ denote the angular frequency and the speed of light in the free space. $\varepsilon_{\mathrm{M}}$ and $\varepsilon_{\mathrm{D}}$ are the complex dielectric functions of a metal film and a dielectric medium. This equation describes a resonance shift resulting from a small change in refractive index induced by interactions amongst biomolecules on the metal surface. Thus, in SPR biosensing, the adsorption of a target analyte by surfaceimmobilized ligands is measured by tracking the change in the resonance condition. Over the past two decades, researchers have performed numerous SPR-based biomolecular interaction analyses to measure biomaterial concentration, thickness, and binding kinetic data for specific biological analytes including antigen/antibody $[4,5]$, protein/protein reactions $[6,7]$, cell responses $[8,9]$, DNA hybridizations $[10,11]$, and other adsorption processes [12].

Gold and silver are the most commonly used as a thin metal film supporting SPPs. While silver films with a narrower SPR curve in the visible band yield higher sensitivity, gold films are employed more frequently because gold is chemically stable and reliable compared with a silver film [13, 14]. On the other hand, it has been known that a surface plasmon wave propagating along the silver surface is less attenuated and exhibits higher localization of electromagnetic field in the dielectric medium than SPPs supported by a

\footnotetext{
*Corresponding author: kmbyun@khu.ac.kr

Color versions of one or more of the figures in this paper are available online.
} 
gold film [2].

There are basically two configurations for SPR biosensing: reflection and transmission types. A conventional SPR biosensor based on the standard Kretschmann configuration works as a reflection-type in the sense that a photodetector measures the reflected light. Its optical platform consists of a light source, an SPR coupler such as a high-index prism or periodic grating surface, and a photodetector on the same side of the light source with respect to the metal film (Fig. 1). For this scheme, the resonance coupling appears as a deep absorption band in the reflectivity of the light spectrum. Depending on the detection method, changes in light intensity at a specific incidence angle, or changes in wavelength or angular position with a minimum reflectance can be measured. Figure 2 shows that refractive index change $\Delta n$ results in the shift of resonance wave-

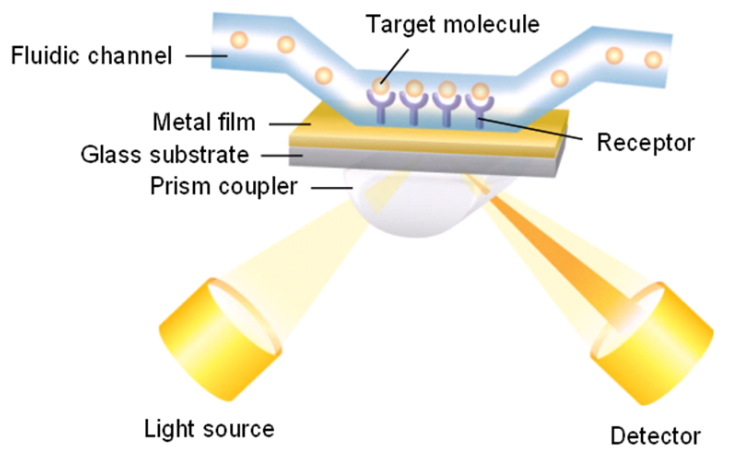

FIG. 1. Schematic of a prism coupler-based reflection-type SPR system. A TM-polarized light with a fixed wavelength or a fixed incidence angle illuminates the sensor substrate through a high-index prism coupler. A thin metal film is deposited on a slide glass with an adhesion layer. Within fluidic channels, receptors are immobilized on the sensor surface and target analytes in an aqueous solution are bound selectively to them.

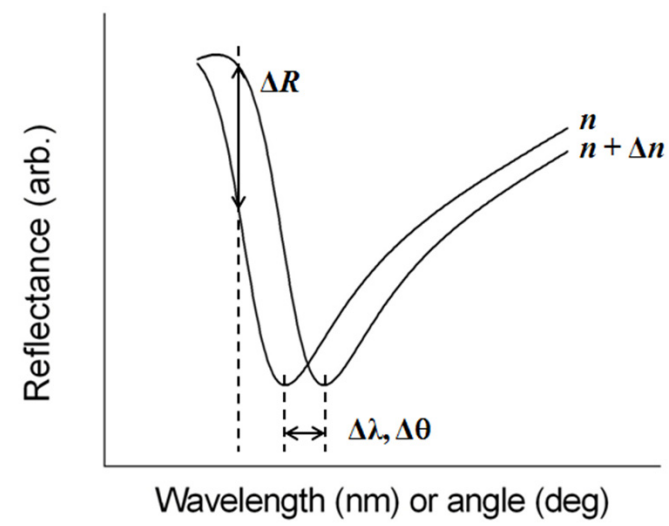

FIG. 2. Typical wavelength or angular spectra of a reflection-type SPR biosensor. The refractive index change $\Delta n$ at the sensor surface leads to the shift in the resonance wavelength $\Delta \lambda$, resonance angle $\Delta \theta$, and the change in the amplitude of reflected light $\Delta R$. length $\Delta \lambda$, angle $\Delta \theta$, and the change in the amplitude of reflected light $\Delta R$ [15].

A transmission-type SPR biosensor, often using a wavelength scanning setup, has also been extensively explored to study spectral outcoupling of surface plasmons excited in metallic nanostructures [16]. As shown in Fig. 3, metallic nanostructures immobilized on a glass substrate are exposed to solvents and aqueous solutions within fluidic channels. The experiment setup uses a white light with normal incidence and the transmitted light is collected by a conventional objective lens and delivered onto a spectrometer via optical fibers.

This configuration is fully based on the localized surface plasmon (LSP) modes. It has been well known that noble metal nanostructures allow direct and strong optical coupling of the incident light to resonantly driven electron plasma oscillations, called localized plasmons [17]. Compared with SPPs excited in a thin metal film, the LSP resonance (LSPR) excitation induces substantial enhancement of electromagnetic fields as a result of strong absorption and highly efficient light scattering. The specific nature of the field enhancement in a metallic nanostructure depends on intrinsic parameters, such as composition, size, and shape, as well as extrinsic factors of the surrounding local media [18-20]. Since spectral position and magnitude of LSPR are sensitive to changes in the local environment caused by molecules adsorbed onto the nanostructures, LSPR devices can serve as transmissionbased biosensors [21]. Figure 4 shows that the position of LSPR red-shifts with an increasing dielectric constant due to the buildup of polarization charges on the dielectric side

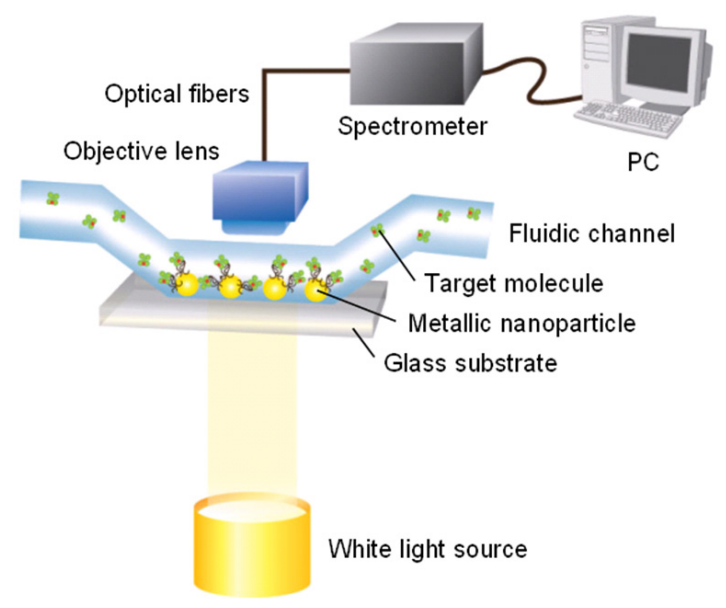

FIG. 3. Schematic of an extinction-based transmission-type LSPR system. Surface-confined metallic nanoparticles are fabricated on a transparent glass substrate in a regular or non-regular manner. UV-visible extinction measurements are collected by a conventional microscope objective and focused onto a fiber-coupled spectrometer. All spectra collected are macroscopic measurements performed in standard transmission geometry using a white light source. The fluidic channel is used to control the external environment of the metallic nanoparticle-based glass substrates. 


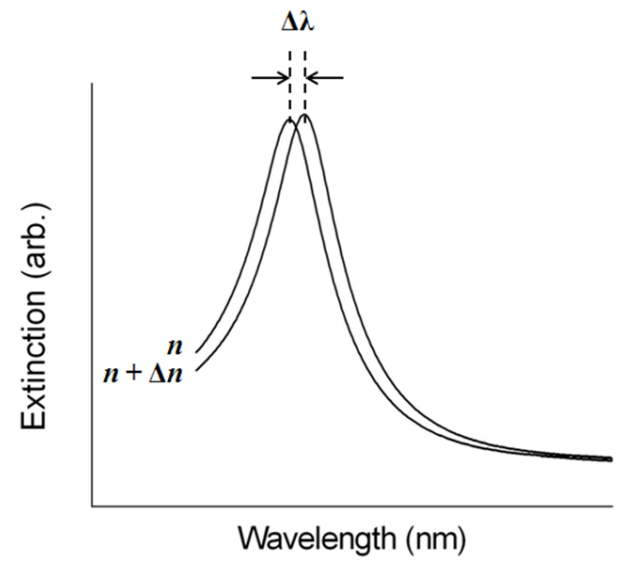

FIG. 4. Typical extinction spectra of a transmission-type LSPR biosensor. The refractive index change $\Delta n$ at the medium surrounding the metallic nanoparticles leads to the shift in the resonance wavelength $\Delta \lambda$.

of the interface [17].

Relative to a conventional SPR, an optical setup to characterize an extinction-based LSPR biosensor is compact and potentially easier to use because it is based on wavelength scanning at normal incidence and allows the light source and the detector to be positioned on opposite sides. A more important characteristic of an LSPR biosensor is its capability for high-throughput monitoring, for example, in DNA research and proteomics for which thousands of binding interactions should be examined rapidly so that individual nanostructure can act as its own transducer [22].

Recent advances in nanoparticle synthesis and nanofabrication techniques have allowed the assembly of complicated patterns of interacting metallic nanostructures. The plasmon resonance of interacting nanostructures leads to interesting and new optical responses obtained from near-field coupling or far-field dipolar interactions depending on the spacing between adjacent nanostructures [23]. For enhanced optical biosensing, the LSPR and its shifts in interacting or noninteracting metallic nanostructures have been extensively exploited. Therefore, this paper reviews optical and physical properties of plasmonic nanostructures and their contributions to a realization of enhanced optical detection platforms, including various LSPR-based approaches for significant amplification of SPR signals.

\section{REFLECTION-TYPE LSPR BIOSENSORS}

\subsection{Colloidal Nanoparticle-Enhanced LSPR Biosensors}

While the traditional metal film-based SPR biosensor has a sensitivity as high as $5 \times 10^{-7}$ refractive index units, which corresponds to $1 \mathrm{pg} / \mathrm{mm}^{2}$ [2, 24], its detection limit can be further improved by coupling the biomolecular recognition of target analyte with another event that leads to larger changes in the SPR signal [25-27]. This signal

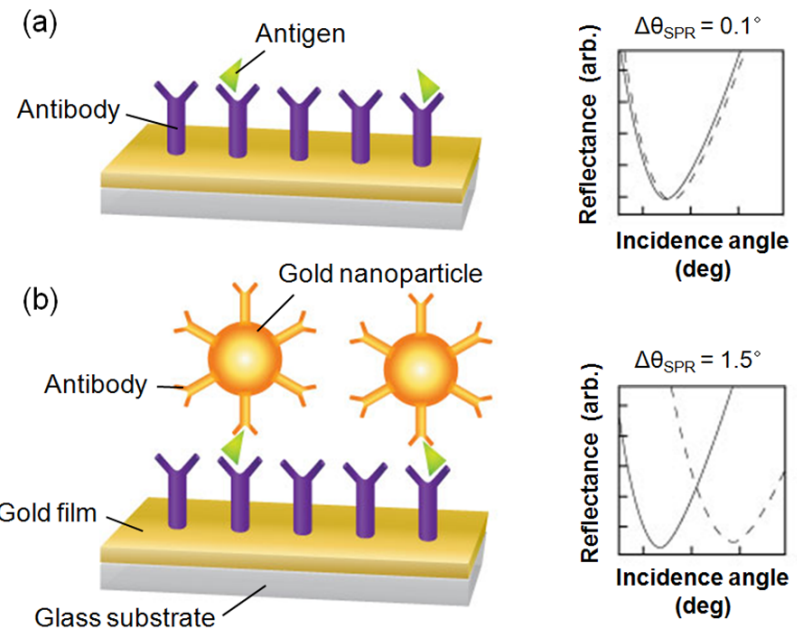

FIG. 5. Schematics and SPR curves of (a) an evaporated gold film modified with a-h-IgG( $\gamma)$ and then exposed to a $1.0 \mathrm{mg} / \mathrm{mL}$ solution of h-IgG and (b) a gold film modified with $a-\mathrm{h}-\operatorname{IgG}(\gamma)$ and then exposed to h-IgG-10-nm gold colloid conjugate [25]. Exposure of an evaporated gold film coated with $\gamma$-chain-specific monoclonal goat anti-human immunoglobulin $\mathrm{G}(a$-h- $\operatorname{IgG}(\gamma))$ to a $1.0 \mathrm{mg} / \mathrm{mL}$ solution of human immunoglobulin $G$ results in a $0.1^{\circ}$ shift in plasmon angle with no change in curve shape (Fig. 5(a)). In contrast, a significantly larger shift of $1.5^{\circ}$, a $2 \%$ increase in minimum reflectance, and noticeable broadening of the curve are observed upon exposure of an identically prepared surface to an electrostatically bound conjugate between $\mathrm{h}$-IgG and 10-nm-diameter colloidal gold [h-IgG-Au] (Fig. 5(b)). On the basis of plasmon shift alone, this represents a 15 -fold increase in SPR sensitivity to the biomolecular interaction event.

enhancement can be achieved by labeling the adsorbed analyte with dielectric or metallic nanoparticles. These labels obviously increase the optical constant in the vicinity of the metal surface and also can induce a plasmonic coupling of LSP modes with propagating SPPs on the flat metal film, resulting in additional SPR shifts. A wide range of nanoparticles have been introduced, including colloidal metallic nanoparticles [28, 29], latex particles [30], and dye-doped polymer particles [31].

As an example of a metallic nanoparticle-enhanced LSPR biosensor, antibody-antigen interaction was measured as shown in Fig. 5. In this experiment, a surface-immobilized antibody is sequentially exposed to antigen and a solution of antibody-conjugated gold nanoparticles [25]. The SPR curves in Fig. 5 show that the shift of SPR angle upon binding the antigen alone is very small compared to the shift observed when the secondary antibody is conjugated with gold nanoparticles. This approach has been also used for detecting DNA hybridization [32]. In a similar way, single-stranded DNA was linked covalently to the gold surface and then hybridized by the complementary DNA, which itself was functionalized by gold nanoparticles. Gold 
nanoparticles attached to the target DNA caused 18-fold signal amplification which was explained by the interaction of localized and propagating surface plasmons. In addition, it was demonstrated that the particle size, material, and surface coverage as well as the metallic substrate can affect the measured signal enhancement [33].

On the other hand, the reflectance characteristics of SPR biosensors employing colloidal metallic nanoparticles are different from those for conventional SPR biosensor, in that they exhibit a broad reflectivity spectrum and an increase in minimum reflectance. For good biosensing performance, a narrow spectral width and a deep absorption band are desired, because with a highly broad and shallow SPR curve, it becomes quite difficult to detect the resonance position accurately and to analyze the effect of binding events on a SPR structure precisely. Moreover, this approach involves complex procedures in the preparation of nano-sized colloidal nanoparticles with functional immobilizations in order to tag the target analytes, and thus it eliminates the important advantage of the SPR technique, namely that labeling is not essential [22].

\subsection{Nanoparticle-Embedded LSPR Biosensors}

The unique properties of metallic nanostructures have attracted significant attention because of the physical characteristics such as local field enhancement and surface-enhanced Raman scattering. It has been demonstrated that the Raman scattering of the underlying molecules can be enhanced by fabricating SPR substrates with metallic nanoparticles [34]. Inspired by this, the colloidal gold nanoparticles are synthesized in a chemical operation and then immobilized on a thin gold film. In Fig. 6, embedding metallic nanostructures on a gold film is an effective way of increasing the roughness of the substrate, which therefore results in a notable

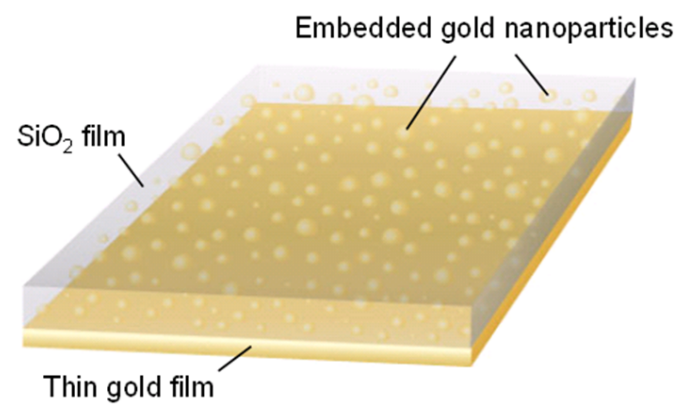

FIG. 6. LSPR sensor substrate with gold nanoparticles embedded in a dielectric layer on a thin gold film [35]. A 47.5-nm thick gold layer was initially deposited on a slide glass using a dc magnetron sputter. Subsequently, mixed thin films composed of $\mathrm{SiO}_{2}$ and gold nanoparticles were deposited via an RF and dc magnetron co-sputtering process. A close inspection of the transmission electron microscopy results revealed that most of the gold nanoparticles with an estimated size of $\sim 4 \mathrm{~nm}$ and spacing between nanoparticles of $\sim 6 \mathrm{~nm}$ were uniformly distributed over the substrate surface. enhancement in the light scattering and absorption.

At the initial stage, researchers introduced a method to immobilize gold nanoparticles on a thin gold film by drying a chemical solution containing suspended gold nanoparticles. However, due to the difficulties in controlling and quantifying the volume fraction of the nanoparticles actually deposited on the film, alternative fabrication methods were developed in which gold nanoclusters were embedded in the dielectric film via an RF magnetron co-sputtering process. Using an SPR substrate with embedded gold nanostructures, DNA hybridization experiments presented an improved detection performance of approximately $0.1 \mathrm{pg} / \mathrm{mm}^{2}$ [35]. This technique provides a feasible and reliable method to control the optimal size and volume fraction of the gold nanoclusters embedded in the dielectric film without the need for complex strategies to amplify the SPR angle shift.

Another example is the work of Matsui et al. on gold nanoparticle-embedded hydrogel as a SPR sensing medium [36]. By swelling of the hydrogel and an increase in the distance between the gold nanoparticles, the medium was functionalized to be selective to the binding of small molecules. An enhanced shift in the SPR curve to higher SPR angles was observed by the interplay of propagating SPPs and resonant LSP modes.

\subsection{Periodic Nanostructure-Based LSPR Biosensors}

With rapid development of nanotechnologies that allow metallic nanostructures to be fabricated and synthesized on the nanometer scale, it has been empirically shown that the use of metallic nanostructures enables an efficient optical coupling of incident light to plasmon resonance, which has been utilized to amplify the optical signals of conventional SPR biosensors. Among various interaction mechanisms between incident light and surface plasmons in play in the sensitivity enhancement, two major mechanisms at work are the SPP-LSP interaction and the LSP-LSP coupling between adjacent nanostructures, resulting in complex resonance properties with an additional shift of SPR angle [37]. In particular, the exploitation of periodic nanostructures can offer the advantage of spatial uniformity and performance reproducibility, while preserving the profit of label-free detection.

As an example of LSPR optical sensing based on periodic nanostructures, one-dimensional (1D) subwavelength gold nanogratings on a thin gold film were explored (Fig. 7(a)). The numerical calculations using rigorous coupled-wave analysis $[38,39]$ and a finite-difference time-domain (FDTD) method have presented interesting LSPR sensing characteristics of 1D nanogratings built on the flat surface. Byun et al. showed that the enhancement enabled by the excitation of LSP modes in gold nanogratings, offers improved performance in sensitivity by more than an order of magnitude compared with a conventional SPR configuration [40]. Optimal gold nanogratings were then designed in terms of the robustness to fabrication errors in implementing periodic structures and the various sensor performances, such as 


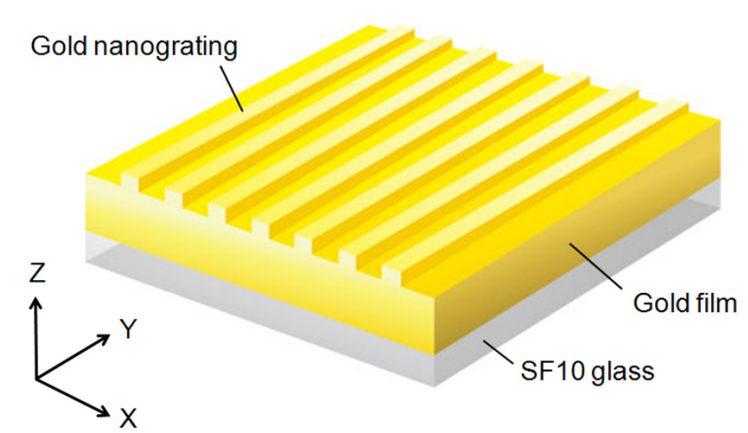

(a)

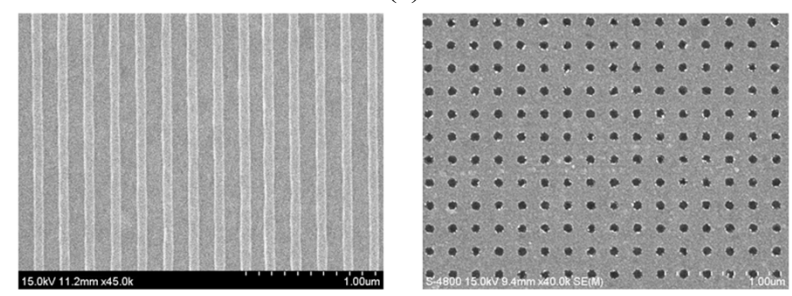

(b)

FIG. 7. (a) Schematic of a gold nanograting-mediated LSPR substrate. 1D gold nanograting is regularly patterned on a thin gold film. (b) Scanning electron micrograph images of $1 \mathrm{D}$ gold nanograting (period $=200 \mathrm{~nm}$ and width $=100 \mathrm{~nm}$, thickness $=20 \mathrm{~nm}$ ) and $2 \mathrm{D}$ gold nanowell array (period $=200 \mathrm{~nm}$, diameter $=80 \mathrm{~nm}$, thickness $=15 \mathrm{~nm}$ ) on a gold surface.

SPR angle shift, SPR curve angular width, and minimum reflectance at resonance [41].

Subsequently, the effect of surface roughness on the sensitivity of gold nanograting-based LSPR biosensors was investigated [42]. Contrary to a conventional scheme with no distinguishable decrease in sensor performance regardless of the correlation length when the roughness $<1 \mathrm{~nm}$, surface roughness displayed a substantial effect on the sensitivity for SPR substrates with gold nanogratings at $\Lambda<200 \mathrm{~nm}$ while the enhancement deteriorated by more than $30 \%$ at a short correlation length less than $100 \mathrm{~nm}$, because the presence of surface fluctuation causes disordered coupling between nanogratings and consequently discourages the enhancement of the sensitivity.

Additionally, the correlation between the localized plasmonic fields induced by the surface nanostructure and the position of target adsorption was analyzed quantitatively and it was found that target localization on nanograting sidewalls improves sensitivity significantly because of strong overlap with resonance LSP modes [43]. To confirm this effect, enhanced electromagnetic fields near the sensor surface were visualized by calculating the spatial distributions of $E_{X}, H_{Y}$, and $E_{Z}$ based on the FDTD method. The minimum grid size for the FDTD was $0.5 \mathrm{~nm}$. Figures 8 and 9 show the field distributions of $\mathrm{E}_{Z}$ for $1 \mathrm{D}$ gold nanogratings with a period of $100 \mathrm{~nm}$, a width of $50 \mathrm{~nm}$, and a thickness of $10 \mathrm{~nm}$. The figures present well-known features of LSPs excited at a metallic nanostructures that are quite typical

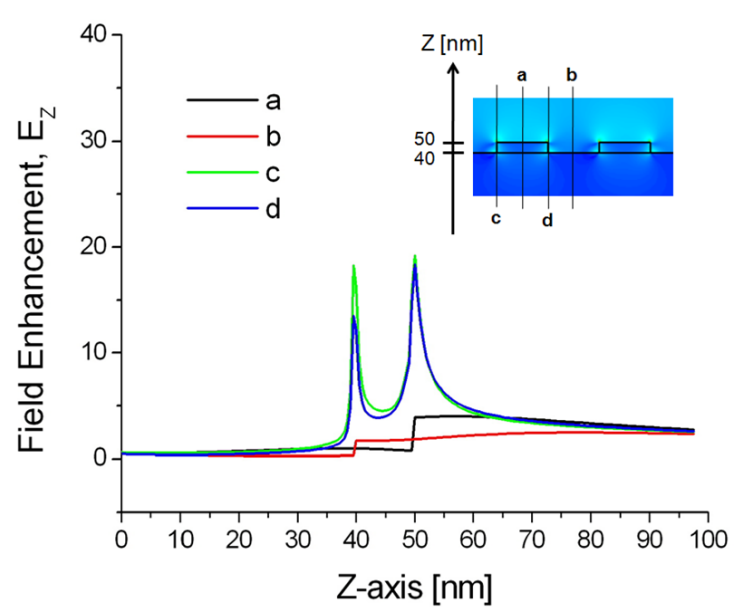

FIG. 8. Vertical field intensity distributions of $E_{Z}$ around the LSPR sensor surface for $1 \mathrm{D}$ nanograting with a period $=100 \mathrm{~nm}$, a width $=50 \mathrm{~nm}$, and a thickness $=10 \mathrm{~nm}$. TM-polarized light with a wavelength of $633 \mathrm{~nm}$ is incident on the substrate. The insets are 2D images obtained from FDTD calculations normalized by the field intensity of 20 .

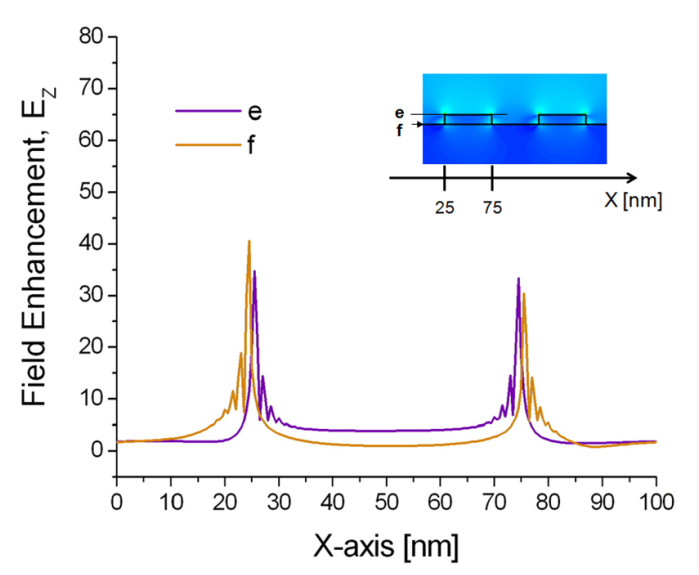

FIG. 9. Horizontal field intensity distributions of $E_{Z}$ around the LSPR sensor surface for $1 \mathrm{D}$ nanograting with a period $=100 \mathrm{~nm}$, a width $=50 \mathrm{~nm}$, and a thickness $=10 \mathrm{~nm}$. TM-polarized light with a wavelength of $633 \mathrm{~nm}$ is incident on the substrate. The insets are 2D images obtained from FDTD calculations normalized by the field intensity of 20 .

of the fields of nonregular metallic nanostructures [44]. On the assumption of an incident electric field of unit amplitude, maximum field amplitudes obtained by FDTD were $\mathrm{E}_{\mathrm{X}}=$ 59, $\mathrm{H}_{\mathrm{Y}}=8$, and $\mathrm{E}_{\mathrm{Z}}=41$. What is as important as field enhancement is where these maxima occur. Obviously, the amplitude of plasmon waves decays rapidly when one moves further away from the nanostructure surface. Since locally enhanced plasmons called "hot spots" are distributed at very short distances from the surface [45, 46], only a limited number of biomolecules within the local fields participate in the resonance shift of an LSPR biosensor, even if a large number of molecules are involved in the interaction. 
The results in Figs. 8 and 9 also indicate that all maxima are located within $1 \mathrm{~nm}$ from the surface in the vicinity of vertices. In addition, because of the field enhancement on nanograting sidewalls, biomolecules on sidewalls participate more vigorously on average than those on nanograting tops and bottoms. As a result, an efficient interplay between target analytes and enhanced LSPs leads to the most prominent improvement of sensitivity with target localization. Note that there are, in fact, multiple peaks associated with each corner. Since the decay length of an LSP resonance from the surface of a metallic nanostructure is longer than the interpeak distance, the multiple peaks of each corner should be regarded as a whole as an individual LSP resonance.

Experimentally, an LSPR substrate with periodic gold nanograting was fabricated as shown in Fig. 7(b) and its sensitivity enhancement was confirmed when evaluated by changing a refractive index of the medium surrounding the sensor substrate. For measuring sensitivity characteristics of subwavelength nanogratings with $\Lambda<300 \mathrm{~nm}$, the nanograting samples were fabricated on a thin gold film by electron beam lithography. Our substrates were spin coated by a $100 \mathrm{~nm}$ thick photoresist film. The regions exposed to an electron beam were chemically dissolved, and the resulting photoresist layer was used as a mask for dry etching of a gold film. Following uniform dry etching, a residual photoresist layer was finally removed by means of a plasma asher. After these processes, we produced 1D gold nanogratings arranged on $40 \mathrm{~nm}$ thick gold/SF10 glass substrates, and we experimentally observed a significant SPR signal amplification. As biological events on the sensor surface, the formation of self-assembled monolayer (SAM) and DNA hybridization were employed and the experimental results showed a significant improvement in sensitivity, well consistent with theoretical predictions [47, 48].

Recently, more complex structures such as two-dimensional (2D) gold nanopost or nanowell arrays regularly patterned on an SPR substrate have been studied to demonstrate highly sensitive label-free detection of DNA hybridization (Fig. 7(b)). Similar to the case of 1D nanograting, the sensitivity enhancement was associated with a surface-limited increase of reaction area and structural perturbation of periodic gold nanostructures on a thin gold film. The perturbation prompts propagating SPPs to interfere with excited LSPs, achieving local field enhancement by resonantly exciting LSP modes and coupling effects between the LSP modes. Experimental data demonstrated that 2D gold nanostructures fabricated on a thin gold film can enhance the sensitivity of conventional SPR biosensor significantly. The data also indicated that both the nanostructure size and period play a role in SPR signal amplification [49].

Since the development of a high-throughput SPR imaging system has emerged as one of the most important biosensing applications, it is thus an obvious next step in an evolutionary path to investigate whether periodic nanostructures can equally enhance the sensitivity for SPR imaging. It was found that periodic gold nanostructures with a narrow width and a small period presented highly enhanced sensitivity when measuring reflected intensity difference with binding analytes from without [50]. Also, the results displayed extremely linear sensing characteristics over a broad range of binding refractive index. On the other hand, the presence of metallic nanostructures may strongly influence the broadening of a SPR curve and a decrease of sensing contrast. Hence, the enhancement obtained from the reflectance change is generally less than for SPR angle shift, while periodic metallic nanostructure-mediated SPR imaging system demonstrated a potential to implement a rapid SPR microarray imaging system with an enhanced sensitivity.

\section{TRANSMISSION-TYPE LSPR BIOSENSORS}

\subsection{Surface-Immobilized Nanostructure-Based LSPR Biosensors}

It has been well known that arrays of gold or silver nanoparticles formed on a transparent substrate exhibit an LSPR extinction band that can be tuned from the near-UV to mid-IR by simply changing the size, shape, and spacing of the nanoparticles $[16,37]$. The extinction peak position is sensitive to the local changes in refractive index occurring near the surface of the immobilized nanoparticles, and the peak position red-shifts linearly with an increasing concentration of target analytes. In particular, the responses of LSPR biosensor vary in a complex and nonlinear fashion when binding events occur at large distances from the surface of the metallic nanoparticles [51].

Theoretically, suppose that we have well-separated and noninteracting nanostructures which are significantly smaller than the incidence wavelength (i.e., $\lambda \gg 2 R$, where $R$ is the radius of the nanoparticle). According to the Mie-scattering theory, the optical extinction of interaction-free spherical metallic nanoparticles can be reduced as follows [17]:

$$
E(\omega)=\frac{9 \omega \varepsilon_{d}{ }^{3 / 2} V}{c}\left\{\frac{\varepsilon_{i}(\omega)}{\left[\varepsilon_{r}(\omega)+2 \varepsilon_{d}\right]^{2}+\varepsilon_{i}(\omega)^{2}}\right\}
$$

$E(\omega)$ is the optical extinction (i.e., sum of absorption and scattering), $\omega$ is the angular frequency of the exciting radiation, $\mathcal{E}_{d}$ is the dielectric function of the binding layer on the nanostructure, $V$ is the nanosphere volume, $\varepsilon_{r}$ and $\varepsilon_{i}$ are the real and imaginary parts of the dielectric function of the metallic nanostructure, and $\mathrm{c}$ is the light velocity in the free space. As presented in Eq. (2), the resonance condition of the LSP is satisfied when $\varepsilon_{r}(\omega)=-2 \mathcal{E}_{d}$ and $\varepsilon_{i}$ is small. Equation 2 does not describe the optical behavior of larger metallic nanoparticles beyond the Rayleigh approximation $(2 \mathrm{R} \geq 30 \mathrm{~nm})$. In those cases, scattering processes and complex plasmonic effects such as retardation and radiative losses start to contribute significantly to the plasmon damping $[52,53]$. In other words, the extinction characteristics are 
dominated by higher-order multipole absorption and scattering, and the full Mie equation needs to be used to model the absorption spectra $[54,55]$. On the other hand, for a nonspherical nanostructure with a noncircular cross section, surface plasmons are not distributed uniformly, manifesting in a shape dependence of the resonance of LSP modes $[56,57]$. Therefore, both the shape and the dielectric function of the nanostructure affect and determine the optical extinction properties.

Figure 10 shows a demonstration of the adsorption-induced LSPR shift obtained from silver nanoparticles immobilized on a glass substrate [58]. Silver nanoparticles have in-plane widths of $\sim 100 \mathrm{~nm}$ and out-of-plane heights of $\sim 50 \mathrm{~nm}$. To prepare the LSPR biosensor for biosensing events, the nanoparticles were first functionalized with a SAM and the LSPR was measured to be $598.6 \mathrm{~nm}$. Next, biotin was covalently attached and its LSPR was determined to be $609.6 \mathrm{~nm}$, corresponding to $11 \mathrm{~nm}$ red-shift. The LSPR biosensor was then exposed to the target analyte of streptavidin (SA) and an exposure to $100 \mathrm{nM}$ SA led to the extinction peak at $636.6 \mathrm{~nm}$, corresponding to an additional $27 \mathrm{~nm}$ shift. As a result, further modification of the sensor substrate by a biotin and SA provokes an additional resonance shift. Also, note that the signal transduction mechanism in this approach is a reliably measured wavelength shift rather than an intensity change as in many previously reported nanoparticle-based transmission-type LSPR biosensors.

Several advantages of a transmission-type LSPR biosensor with plasmonic nanostructures include (1) its compact optical setup which is more appropriate for miniaturization than reflection-type SPR detection, (2) a high sensitivity, especially when the resonance of the analyte combined with various labels overlaps with the intrinsic LSPR of the metallic nanostructures [59], and (3) an ultrahigh spatial
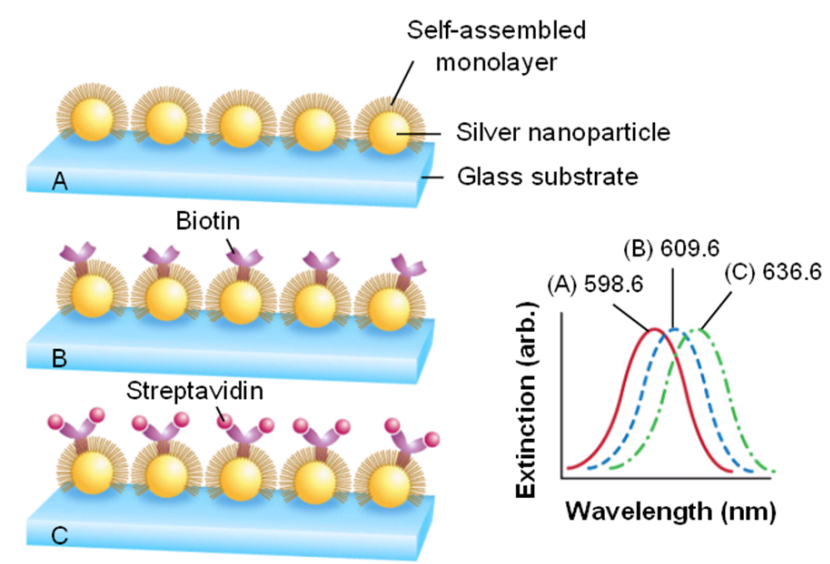

FIG. 10. Detection of SA binding to biotinylated silver nanoparticles immobilized on a glass substrate. (A) Silver nanoparticles after surface modification with $1 \mathrm{mM}$ SAM, $\lambda_{\text {LSPR }}=598.6 \mathrm{~nm}$. (B) Silver nanoparticles after modification with $1 \mathrm{mM}$ biotin, $\lambda_{\mathrm{LSPR}}=609.6 \mathrm{~nm}$. (C) Silver nanoparticles after modification with $100 \mathrm{nM} \mathrm{SA}, \lambda_{\text {LSPR }}$ $=636.6 \mathrm{~nm}$. resolution because a single nanoparticle can act as an individual transducer for precious or limited-volume samples. As an example, a LSPR shift of $\sim 40 \mathrm{~nm}$ was measured from Rayleigh scattering spectroscopy for specific binding in low concentration of $\sim 100$ zeptomoles by employing a single triangular silver nanoparticle [60].

In terms of formation of metallic nanostructures immobilized on a glass substrate, various synthesis and fabrication techniques have been investigated. One of the commonly used procedures for yielding spherical nanoparticles is the reduction of metal salts in a solution containing a specific stabilizer, such as ligands, surfactants, or polymers, which binds to the surface of nanoparticles and inhibits their aggregation [61]. The size and shape of nanoparticles can be adjusted by the reductant, stabilizer, and concentrations of reagents. More complex nanostructures nanorods or nanoshells can be prepared by seeding growth techniques [62-64]. The morphology of a metallic nanorod with two distinct plasmon modes associated with its non-symmetric shape is determined by the composition of seeds, surfactant, and additives. For example, in Fig. 11, gold nanorods were synthesized electrochemically in the presence of a surfactant and cosurfactant solution mixture known to form rodshape micelles $[65,66]$. The shape distribution of the rods depends on the current and the ratio of the concentration of the surfactant to that of the cosurfactant used. Moreover, by metalizing colloidal silica nanosphere cores, gold nanoshells can be obtained and the final thickness of gold shell is controlled by changing the initial gold seed coverage and the concentration of reductant used in the electroless plating process [67]. On the other hand, in order to improve the reliability of the nanostructured substrate and to control the size, shape, and spacing of metallic nanostructures precisely, lithographic techniques such as electron beam lithography and focused ion beam lithography have been used frequently [48, 49, 68]. After patterning thin resist layer

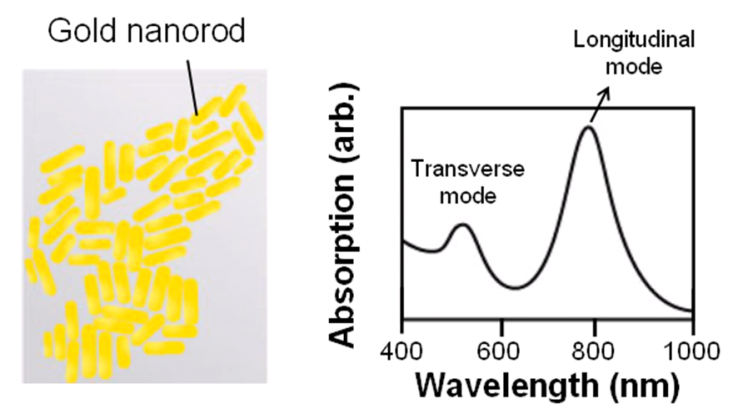

FIG. 11. Synthesis of gold nanorods and their absorption spectrum. Two distinct plasmon modes become more pronounced when the aspect ratio of the metallic nanorods is increased: one at a shorter wavelength of $\sim 520 \mathrm{~nm}$ is due to the coherent electron oscillation along the short axis, and the other is at a longer wavelength of $\sim 780 \mathrm{~nm}$, which is more intense and obtained from the coherent electron oscillation along the long axis [66]. 
with a high power scanning beam, the patterned resist is utilized as a sacrificial masking layer in subsequent etching or metal deposition processes, resulting in well-controlled $1 \mathrm{D}$ or $2 \mathrm{D}$ nanostructures.

It is also interesting that unconventional approaches, which are capable of patterning large areas at a relatively low cost, provide an effective alternative to scanning beam lithographic techniques. For example, triangular nanoparticle arrays obtained from hexagonally distributed colloidal crystal mask were fabricated by using nanosphere lithography [69]. Soft lithography is another well-known versatile technique for patterning periodic metallic structures at the nanoscale over large areas [70]. These conventional and unconventional fabrication methods are practical for both reflection and transmission-type LSPR biosensing configurations.

\subsection{Extraordinary Optical Transmission-Based LSPR Biosensors}

From the experiments on the light transmission through arrays of nanoholes in thin gold and silver films, it was found that the amount of transmitted light at certain wavelengths was much greater than predicted by the classical aperture theory; i.e., the substrate appeared much more transparent than it should be [71, 72]. This unexpected result is called extraordinary optical transmission. In principle, the peaks of maximum transmission were dependent on the period between the nanoholes. The excitation of surface plasmons seems to mainly contribute to the effect of extraordinary optical transmission, while its interpretation is still underway [73].

The transmission characteristics through arrays of nanoholes are related to the optical properties of the metal-dielectric interface. At normal incidence, the resonance wavelength $\left(\lambda_{\text {peak }}\right)$ from nanohole arrays with symmetric square lattice can be estimated by Eq. (3),

$$
\lambda_{\text {peak }}(i, j)=\frac{p \sqrt{\frac{\varepsilon_{\mathrm{M}} \varepsilon_{\mathrm{D}}}{\varepsilon_{\mathrm{M}}+\varepsilon_{\mathrm{D}}}}}{\sqrt{i^{2}+j^{2}}}
$$

where $p$ is the lattice constant, $i$ and $j$ denote the scattering orders of the array, and $\varepsilon_{\mathrm{M}}$ and $\varepsilon_{\mathrm{D}}$ are the dielectric constants of the metal and its adjacent medium, respectively [71]. Therefore, the adsorption of biomolecules on the metallic substrate may induce a shift of resonance wavelength or a change in peak intensity. In contrast to the conventional reflection-type SPR biosensor, the momentum matching condition for plasmon resonance in zero-order transmission is given by the periodicity of the nanoholes and thus, prism coupling is not required [74].

Figure 12 demonstrates an experimental setup to measure the extraordinary optical transmission effect $[75,76]$. The metal film is deposited on a glass slide and the array is fabricated using focused ion beam milling. The transmission spectra are obtained using a spectrometer via an optical

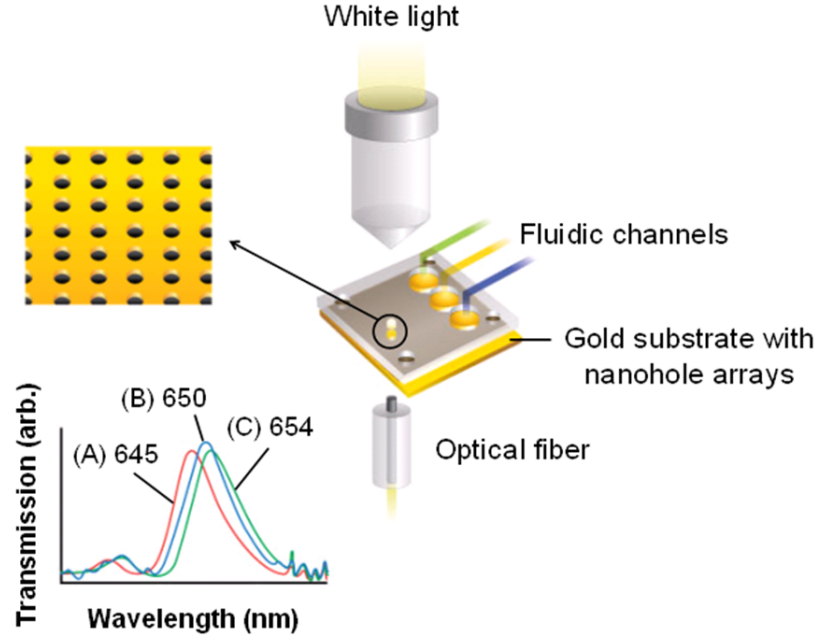

FIG. 12. Experimental setup for light transmission measurements through the arrays of subwavelength holes and transmission spectra of normally incident white light [76]. All spectra were obtained using near-IR source from the halogen lamp, objective lens $(\times 100)$, and optical fiber-coupled spectrometer. The arrays of subwavelength holes were fabricated using focused ion beam milling and the diameter of the nanohole arrays was $200 \mathrm{~nm}$ and the gold film was 100-nm thick. In biological experiments, the substrates were modified with a SAM and then were immersed in $100 \mu \mathrm{M}$ aqueous solution of bovine serum albumin (BSA). The transmission peaks were found at (A) $645 \mathrm{~nm}$ for bare gold surface, (B) $650 \mathrm{~nm}$ for gold substrate modified with a SAM, and (C) $654 \mathrm{~nm}$ for BSA adsorption to the SAM immobilized on the gold surface, respectively.

fiber coupler. Peaks in the white light transmission correspond to the wavelengths that match the conditions of Eq. (3) for the changes in the dielectric properties of the surface. The normal incidence setup allied to the small footprint of nanohole arrays offers an attractive opportunity for miniaturization. From the experiments in Fig. 12, the sensor sensitivity with an order of magnitude better than the values from angle-resolved SPR systems was obtained. It is important to point out that the sensing area of the nanohole arrays is highly localized and hence, the changes in both peak positions and intensities originate from a smaller number of target molecules. This new approach for SPR sensing is more compatible with the lab-on-chip concept and provides the possibility of high-throughput analysis from a single SPR chip [74, 77].

\section{FUTURE DIRECTION AND PROSPECTS}

Beyond the pursuit of ultrahigh sensitivity for enhanced detection of biomolecules at a very low concentration, recent trends are focused on two representative topics: robust integration and miniaturization for portable biosensors and development of sensor platforms for high-throughput screening. 
Most of commercial SPR biosensor systems are generally based on the conventional prism-coupled SPR configuration with angle interrogation scheme. Although this approach is simple, robust, and sensitive, it is not in practice appropriate for miniaturization. Hence, there are continuing efforts to employ optical fibers and waveguide structures as an alternative to the prism-based configuration. After an initial exploitation of single mode optical fibers which were sidepolished and coated with a thin metal film [78], the fiber tip with a form of micro-prism has been optimized to improve the coupling of light to SPP modes and a polarization-maintaining fiber-based SPR sensing has been developed for stable polarization control and accurate measurement $[79,80]$.

Another alternative approach has been the planar optical waveguides with a multilayer structure. These waveguides may realize the development of portable SPR biosensor systems because they enable accommodation of multiple sensing elements on a single substrate using micro-fabrication technology [81]. In particular, by designing multimode waveguides in which a polychromatic radiation is incident on the different sensing channels at different angles of incidence or on a high refractive index dielectric overlayer which shifts the resonance wavelength for a part of the sensing surface, it is possible to extract information of target analytes on the molecular density and on the refractive index on the sensing layer simultaneously and thus to analyze the complex samples quantitatively [82]. Moreover, a miniaturized SPR platform based on optical fibers or planar waveguides can be combined with plasmonic nanostructures to recognize the adsorbed biomolecules more efficiently. With an additional layer involving metallic nanostructures placed on a thin metal film, an improved sensitivity to the local index change could be accomplished.

In terms of high-throughput capability, SPR imaging in which a collimated light is incident on a sensor surface with a fixed angle has been the strongest candidate for microarray-based multi-channel detection among reflection-type SPR biosensors. Its distinguishing feature is the use of CCD camera where the SPR signals are captured as images after passing through a narrow band interference filter [83]. While SPR imaging scheme has an important advantage of simplicity of structure with no moving parts, the intensity-based method has difficulties in achieving significant detection accuracy because of insufficient sensitivity. In order to surmount this restriction on sensitivity, an introduction of metallic nanostructures in SPR imaging biosensors has been investigated [26, 50]. The sensitivity improvement in SPR imaging can be slightly degraded due to the broadening of a SPR curve and a decrease of sensing contrast, accompanied by the presence of metallic nanostructures. Nevertheless, rapid development of nanofabrication techniques will make it possible to realize an enhanced SPR imaging with high-density arrays and high sensitivity for future genomic and proteomic works. Another interesting approach for SPR imaging is an interferometry-based phase detection system producing an interference pattern on a CCD camera [84]. While it has a small dynamic range and limitations in facilitating real time SPR sensing, interferometry may produce more rapid changes than intensity, demonstrating the potential for a sensitive SPR detection with no demand for plasmonic nanostructures.

For transmission-type LSPR biosensors, the transparent sensor substrates incorporating metallic nanostructures are more amenable to miniaturization than reflection-type SPR detection. Also, their sensitivity is known to be comparable to the performance of commercial SPR systems. In particular, as an LSPR biosensing has been demonstrated at the single nanoparticle level using various kinds of metallic nanostructures, an individual or ensembles of nanostructures can work as a single transducer that transforms binding events into corresponding optical changes. In short, since the planar format and transmission optics are suitable for multiplexing in a microarray platform, microarrays for multiple detection and high-throughput analysis seem to be the next step for enhanced transmission-type LSPR biosensors.

\section{CONCLUSION}

In this review, exploitation of plasmonic nanostructures exhibited significant advantages over conventional SPR sensing schemes. Surface plasmons, surface-bound electromagnetic fields, are categorized into two classes of SPPs and LSPs. In reflection-type LSPR biosensors, both classes are present and their interplay contributes to the enhancement of sensing performances, while only LSP modes dominantly affect the sensor characteristics of transmissiontype biosensors. In both cases, however, a plasmonic approach based on nanostructured metallic substrates offers an exciting and promising alternative to surmount the limitations of conventional SPR sensors. Hence, the manipulation of optical properties of surface plasmons by adjusting the geometric parameters of the nanostructures has been the hot issue in the area of plasmonic-based biosensors. Beyond the scope of optical biosensors, the potential for the development of plasmonic devices in the subwavelength regime will be a prominent impetus in several encouraging applications, for instance, plasmonics integrated circuits allowing information processing at an ultrafast speed, superlensing phenomenon surpassing the diffraction limit in imaging systems, and plasmon-assisted nanolithography technique utilizing the confinement of locally enhanced fields.

Especially, it should be emphasized that, to achieve a breakthrough for a full realization of the potential of SPR biosensors, a greater collaboration in a variety of fields is very much required. Together with a better understanding of the underlying fundamentals and a development of theoretical analysis algorithms and new biosensing concepts, advances in the areas of such as opto-electronics for low-noise light 
sources and efficient photodetectors, integrated optics for miniaturizing SPR components and planar waveguides, surface chemistry for effective surface modification and ligand design, microfluidics, and nanofabrication techniques are all essential for the development of point-of-care and in-field SPR biosensors with an ultrahigh sensitivity and selectivity. Relevant in-depth studies are currently under way in many research groups and in the near future, it is hoped that mobile SPR platforms will be in widespread use, satisfying the various needs of the consumers.

\section{ACKNOWLEDGMENT}

This work was supported by Korea Science and Engineering Foundation (KOSEF) grant funded by the Korean government (2010-0017105).

\section{REFERENCES}

1. B. Rothenhäusler and W. Knoll, "Surface-plasmon microscopy," Nature 332, 615-617 (1988).

2. J. Homola, S. S. Yee, and G. Gauglitz, "Surface plasmon resonance sensors: review," Sens. Actuators B 54, 3-15 (1999).

3. H. Raether, Surface Plasmon on Smooth and Rough Surfaces and on Gratings (Springer-Verlag, Berlin, Germany, 1988).

4. M. Malmqvist, "Surface plasmon resonance for detection and measurements of antibody-antigen affinity and kinetics," Curr. Opin. Immunol. 5, 282-286 (1993).

5. T. Akimoto, S. Sasaki, K. Ikebukuro, and I. Karube, "Effect of incident angle of light on sensitivity and detection limit for layers of antibody with surface plasmon resonance spectroscopy," Biosens. Bioelectron. 15, 355-362 (2000).

6. B. Johnsson, S. Löfås, and G. Lindquist, "Immobilization of proteins to a carboxymethyldextran-modified gold surface for biospecific interaction analysis in surface plasmon resonance sensors," Anal. Chem. 198, 268-277 (1991).

7. R. Karlsson and A. Fält, "Experimental design for kinetic analysis of protein-protein interactions with surface plasmon resonance biosensors," J. Immunol. Methods 200, 121-133 (1997).

8. A. L. Plant, M. Brigham-Burke, E. C. Petrella, and D. J. O'Shannessy, "Phospholipid/alkanethiol bilayers for cellsurface receptor studies by surface plasmon resonance," Anal. Biochem. 226, 342-348 (1995).

9. S. A. Kim, K. M. Byun, J. Lee, J. H. Kim, D.-G. A. Kim, H. Baac, M. L. Shuler, and S. J. Kim, "Optical measurement of neural activity using surface plasmon resonance," Opt. Lett. 33, 914-916 (2008).

10. B. P. Nelson, T. E. Grimsrud, M. R. Liles, R. M. Goodman, and R. M. Corn, "Surface plasmon resonance imaging measurements of DNA and RNA hybridization adsorption onto DNA microarrays," Anal. Chem. 73, 1-7 (2001).

11. S. A. Kim, S. J. Kim, S. H. Lee, T. H. Park, K. M. Byun, S. G. Kim, and M. L. Shuler, "Detection of avian influenzaDNA hybridization using wavelength-scanning surface plasmon resonance biosensor," J. Opt. Soc. Korea 13, 392-397 (2009).
12. B. Liedberg, C. Nylander, and I. Lundstrom, "Biosensing with surface plasmon resonance - how it all started," Biosens. Bioelectron. 10, 1-4 (1995).

13. X.-M. Zhu, P.-H. Lin, P. Ao, and L. B. Sorensen, "Surface treatments for surface plasmon resonance biosensors," Sens. Actuators B 84, 106-112 (2002).

14. H. Libardi and H. P. Grieneisen, "Guided-mode resonance absorption in partly oxidized thin silver films," Thin Solid Films 333, 82-87 (1998).

15. M. Piliarik and J. Homola, "Surface plasmon resonance sensors: approaching their limits?," Opt. Exp. 17, 1650516517 (2009).

16. E. Hutter and J. H. Fendler, "Exploitation of localized surface plasmon resonance," Adv. Mater. 16, 1685-1706 (2004).

17. U. Kreibig and M. Vollmer, Optical Properties of Metal Clusters (Springer-Verlag, Berlin, Germany, 1995).

18. S. Underwood and P. Mulvaney, "Effect of the solution refractive index on the color of gold colloids," Langmuir 10, 3427-3430 (1994).

19. P. Mulvaney, "Surface plasmon spectroscopy of nanosized metal particles," Langmuir 12, 788-800 (1996).

20. K. L. Kelly, E. Coronado, L. L. Zhao, and G. C. Schatz, "The optical properties of metal nanoparticles: the influence of size, shape, and dielectric environment," J. Phys. Chem. B 107, 668-677 (2003).

21. J. Zhao, X. Zhang, C. R. Yonzon, A. J. Haes, and R. P. van Duyne, "Localized surface plasmon resonance biosensors," Nanomedicine 1, 219-228 (2006).

22. X. D. Hoa, A. G. Kirk, and M. Tabrizian, "Towards integrated and sensitive surface plasmon resonance biosensors: a review of recent progress," Biosens. Bioelectron. 23, 151-160 (2007).

23. W. Rechberger, A. Hohenau, A. Leitner, J. R. Krenn, B. Lamprecht, and F. R. Aussenegg, "Optical properties of two interacting gold nanoparticles," Opt. Comm. 220, 137-141 (2003).

24. E. Stenberg, B. Persson, H. Roos, and C. Urbaniczky, "Quantitative determination of surface concentration of protein with surface plasmon resonance using radiolabeled proteins," J. Colloid. Interf. Sci. 143, 513-526 (1991).

25. L. A. Lyon, M. D. Musick, and M. J. Natan, "Colloidal Auenhanced surface plasmon resonance immunosensing," Anal. Chem. 70, 5177-5183 (1998).

26. Y. Li, A. W. Wark, H. J. Lee, and R. M. Corn, "Singlenucleotide polymorphism genotyping by nanoparticle-enhanced surface plasmon resonance imaging measurements of surface ligation reactions," Anal. Chem. 78, 3158-3164 (2006).

27. J. S. Mitchell, Y. Wu, C. J. Cook, and L. Main, "Sensitivity enhancement of surface plasmon resonance biosensing of small molecules," Anal. Biochem. 343, 125-135 (2005).

28. X. Liu, Y. Sun, D. Song, Q. Zhang, Y. Tian, S. Bi, and H. Zhang, "Sensitivity-enhancement of wavelength-modulation surface plasmon resonance biosensor for human complement factor 4," Anal. Biochem. 333, 99-104 (2004).

29. L. A. Lyon, M. D. Musick, P. C. Smith, B. D. Reiss, D. J. Peña, and M. J. Natan, "Surface plasmon resonance of colloidal Au-modified gold films," Sens. Actuators B 54, 118-124 (1999).

30. E. F. A. de Vries, R. B. M. Schasfoort, J. van der Plas, and J. Greve, "Nucleic acid detection with surface plasmon 
resonance using cationic latex," Biosens. Bioelectron. 9, 509-514 (1994).

31. E. Fujii, T. Koike, K. Nakamura, S. Sasaki, K. Kurihara, D. Citterio, Y. Iwasaki, O. Niwa, and K. Suzuki, "Application of an absorption-based surface plasmon resonance principle to the development of SPR ammonium ion and enzyme sensors," Anal. Chem. 74, 6106-6110 (2002).

32. L. He, M. D. Musick, S. R. Nicewarner, F. G. Salinas, S. J. Benkovic, M. J. Natan, and C. D. Keating, "Colloidal Au-enhanced surface plasmon resonance for ultrasensitive detection of DNA hybridization," J. Am. Chem. Soc. 122, 9071-9077 (2000).

33. E. Hutter, J. H. Fendler, and D. Roy, "Surface plasmon resonance studies of gold and silver nanoparticles linked to gold and silver substrates by 2-aminoethanethiol and 1,6hexanedithiol," J. Phys. Chem. B 105, 11159-11168 (2001).

34. T. Zhu, X. Zhang, J. Wang, X. Fu, and Z. Liu, "Assembling colloidal $\mathrm{Au}$ nanoparticles with functionalized self-assembled monolayers," Thin Solid Films 327-329, 595-598 (1998).

35. W. P. Hu, S.-J. Chen, K.-T. Huang, J. H. Hsu, W. Y. Chen, G. L. Chang, and K.-A. Lai, "A novel ultrahigh-resolution surface plasmon resonance biosensor with an Au nanoclusterembedded dielectric film," Biosens. Bioelectron. 19, 1465-1471 (2004).

36. J. Matsui, K. Akamatsu, N. Hara, D. Miyoshi, H. Nawafune, K. Tamaki, and N. Sugimoto, "SPR sensor chip for detection of small molecules using molecularly imprinted polymer with embedded gold nanoparticles," Anal. Chem. 77, 4282-4285 (2005).

37. M. E. Stewart, C. R. Anderton, L. B. Thompson, J. Maria, S. K. Gray, J. A. Rogers, and R. G. Nuzzo, "Nanostructured plasmonic sensors," Chem. Rev. 108, 494-521 (2008).

38. M. G. Moharam and T. K. Gaylord, "Rigorous coupled-wave analysis of metallic surface-relief gratings," J. Opt. Soc. Am. A 3, 1780-1787 (1986).

39. L. Li and C. W. Haggans, "Convergence of the coupledwave method for metallic lamellar diffraction gratings," J. Opt. Soc. Am. A 10, 1184-1189 (1993).

40. K. M. Byun, S. J. Kim, and D. Kim, "Design study of highly sensitive nanowire-enhanced surface plasmon resonance biosensors using rigorous coupled wave analysis," Opt. Exp. 13, 3737-3742 (2005).

41. K. M. Byun, D. Kim, and S. J. Kim, "Investigation of the profile effect on the sensitivity enhancement of nanowiremediated localized surface plasmon resonance biosensors," Sens. Actuators B 117, 401-407 (2006).

42. K. M. Byun, S. J. Yoon, D. Kim, and S. J. Kim, "Sensitivity analysis of a nanowire-based surface plasmon resonance biosensor in the presence of surface roughness," J. Opt. Soc. Am. A 24, 522-529 (2007).

43. K. M. Byun, S. M. Jang, S. J. Kim, and D. Kim, "Effect of target localization on the sensitivity of a localized surface plasmon resonance biosensor based on subwavelength metallic nanostructures,” J. Opt. Soc. Am. A 26, 1027-1034 (2009).

44. J. P. Kottmann, O. J. F. Martin, D. R. Smith, and S. Schultz, "Plasmon resonances of silver nanowires with a nonregular cross section," Phys. Rev. B 64, 235402 (2001).

45. L. Qin, S. Zou, C. Xue, A. Atkinson, G. C. Schatz, and C. A. Mirkin, "Designing, fabricating, and imaging Raman hot spots,” Proc. Natl. Acad. Sci. U.S.A. 103, 13300-13303 (2006).
46. E. Hao and G. C. Schatz, "Electromagnetic fields around silver nanoparticles and dimers," J. Chem. Phys. 120, 357-366 (2004).

47. K. M. Byun, S. J. Yoon, D. Kim, and S. J. Kim, "Experimental study of sensitivity enhancement in surface plasmon resonance biosensors by use of periodic metallic nanowires," Opt. Lett. 32, 1902-1904 (2007).

48. K. Kim, D. J. Kim, S. Moon, D. Kim, and K. M. Byun, "Localized surface plasmon resonance detection of layered biointeractions on metallic subwavelength nanogratings," Nanotechnology 20, 315501 (2009).

49. L. Malic, B. Cui, T. Veres, and M. Tabrizian, "Enhanced surface plasmon resonance imaging detection of DNA hybridization on periodic gold nanoposts," Opt. Lett. 32, 3092-3094 (2007).

50. K. M. Byun, M. L. Shuler, S. J. Kim, S. J. Yoon, and D. Kim, "Sensitivity enhancement of surface plasmon resonance imaging using periodic metallic nanowires," IEEE J. Lightwave Technol. 26, 1472-1478 (2008).

51. A. J. Haes, S. Zou, G. C. Schatz, and R. P. van Duyne, "A nanoscale optical biosensor: the long range distance dependence of the localized surface plasmon resonance of noble metal nanoparticles," J. Phys. Chem. B 108, 109-116 (2004).

52. M. Meier and A. Wokaun, "Enhanced fields on large metal particles: dynamic depolarization," Opt. Lett. 8, 581-583 (1983).

53. A. Wokaun, J. P. Gordon, and P. F. Liao, "Radiation damping in surface-enhanced Raman scattering," Phys. Rev. Lett. 48, 957-960 (1982).

54. C. F. Bohren and D. R. Huffman, Absorption and Scattering of Light by Small Particles (John Wiley \& Sons, Inc., New York, USA, 1998).

55. S. Link and M. A. El-Sayed, "Shape and size dependence of radiative, non-radiative and photothermal properties of gold nanocrystals," J. Phys. Chem. B 103, $4212-4217$ (1999).

56. J. P. Kottmann, O. J. F. Martin, D. R. Smith, and S. Schultz, "Spectral response of plasmon resonant nanoparticles with a non-regular shape," Opt. Exp. 6, 213-219 (2000).

57. E. Hao, R. C. Bailey, G. C. Schatz, J. T. Hupp, and S. Li, "Synthesis and optical properties of "Branched" gold nanocrystals," Nano Lett. 4, 327-330 (2004).

58. A. J. Haes and R. P. van Duyne, "A nanoscale optical biosensor: sensitivity and selectivity of an approach based on the localized surface plasmon resonance spectroscopy of triangular silver nanoparticles," J. Am. Chem. Soc. 124, 1059610604 (2002).

59. J. Zhao, A. Das, X. Zhang, G. C. Schatz, S. G. Sligar, and R. P. van Duyne, "Resonance surface plasmon spectroscopy: low molecular weight substrate binding to cytochrome P450," J. Am. Chem. Soc. 128, 11004-11005 (2006).

60. A. D. McFarland and R. P. van Duyne, "Single silver nanoparticles as real-time optical sensors with zeptomole sensitivity," Nano Lett. 3, 1057-1062 (2003).

61. A. Roucoux, J. Schulz, and H. Patin, "Reduced transition metal colloids: a novel family of reusable catalysts?," Chem. Rev. 102, 3757-3778 (2002).

62. Y. Xiong, H. Cai, B. J. Wiley, J. Wang, M. J. Kim, and Y. Xia, "Synthesis and mechanistic study of palladium nanobars and nanorods," J. Am. Chem. Soc. 129, 3665-3675 
(2007)

63. N. R. Jana, L. Gearheart, and C. J. Murphy, "Seed-mediated growth approach for shape-controlled synthesis of spheroidal and rod-like gold nanoparticles using a surfactant template," Adv. Mater. 13, 1389-1393 (2001).

64. A. E. Neeves and M. H. Birnboim, "Composite structures for the enhancement of nonlinear-optical susceptibility," J. Opt. Soc. Am. B 6, 787-796 (1989).

65. Y.-Y. Yu, S.-S. Chang, C.-L. Lee, and C. R. C. Wang, "Gold nanorods: electrochemical synthesis and optical properties," J. Phys. Chem. B 101, 6661-6664 (1997).

66. M. A. El-Sayed, "Some interesting properties of metals confined in time and nanometer space of different shapes," Acc. Chem. Res. 34, 257-264 (2001).

67. S. J. Oldenburg, R. D. Averitt, S. L. Westcott, and N. J. Halas, "Nanoengineering of optical resonances," Chem. Phys. Lett. 288, 243-247 (1998).

68. T. Ohno, J. A. Bain, and T. E. Schlesinger, "Observation of geometrical resonance in optical throughput of very small aperture lasers associated with surface plasmons," J. Appl. Phys. 101, 083107 (2007).

69. C. L. Haynes and R. P. van Duyne, "Nanosphere lithography: a versatile nanofabrication tool for studies of sizedependent nanoparticle optics," J. Phys. Chem. B 105, 5599-5611 (2001).

70. J. A. Rogers and R. G. Nuzzo, "Recent progress in soft lithography," Mater. Today 8, 50-56 (2005).

71. T. W. Ebbesen, H. J. Lezec, H. F. Ghaemi, T. Thio, and P. A. Wolff, "Extraordinary optical transmission through sub-wavelength hole arrays," Nature 391, 667-669 (1998).

72. C. Genet and T. W. Ebbesen, "Light in tiny holes," Nature 445, 39-46 (2007).

73. H. Gao, J. Henzie, and T. W. Odom, "Direct evidence for surface plasmon-mediated enhanced light transmission through metallic nanohole arrays," Nano Lett. 6, 2104-2108 (2006).

74. R. Gordon, D. Sinton, K. L. Kavanagh, and A. G. Brolo, "A new generation of sensors based on extraordinary optical transmission," Acc. Chem. Res. 41, 1049-1057 (2008).

75. A. de Leebeeck, L. K. S. Kumar, V. de Lange, D. Sinton, R. Gordon, and A. G. Brolo, "On-chip surface-based detection with nanohole arrays," Anal. Chem. 79, 4094-4100 (2007).

76. A. G. Brolo, R. Gordon, B. Leathem, and K. L. Kavanagh, "Surface plasmon sensor based on the enhanced light transmission through arrays of nanoholes in gold films," Langmuir 20, 4813-4815 (2004).

77. P. R. H. Stark, A. E. Halleck, and D. N. Larson, "Short order nanohole arrays in metals for highly sensitive probing of local indices of refraction as the basis for a highly multiplexed biosensor technology," Methods 37, 37-47 (2005).

78. J. Homola, "Optical fiber sensor based on surface plasmon resonance excitation," Sens. Actuators B 29, 401-405 (1995).

79. K. Kurihara, H. Ohkawa, Y. Iwasaki, O. Niwa, T. Tobita, and K. Suzuki, "Fiber-optic conical microsensors for surface plasmon resonance using chemically etched single-mode fiber," Anal. Chim. Acta 523, 165-170 (2004).

80. M. Piliarik, J. Homola, Z. Maníková, and J. Čtyroký, "Surface plasmon resonance sensor based on a single-mode polarization-maintaining optical fiber," Sens. Actuators B 90, 236-242 (2003).

81. J. Homola, "Present and future of surface plasmon resonance biosensors," Anal. Bioanal. Chem. 337, 528-539 (2003).

82. P. Stöcker, B. Menges, U. Langbein, and S. Mittler, "Multimode waveguide mode surface plasmon coupling: a sensitivity and device realizability study," Sens. Actuators A 116, 224-231 (2004).

83. C. E. Jordan, A. G. Frutos, A. J. Thiel, and R. M. Corn, "Surface plasmon resonance imaging measurements of DNA hybridisation adsorption and streptavidin/DNA multilayer formation at chemically modified gold surfaces," Anal. Chem. 69, 4939-4947 (1997).

84. A. V. Kabashin and P. Nikitin, "Surface plasmon resonance interferometer for bio- and chemical-sensors," Opt. Comm. 150, 5-8 (1998). 